\title{
Detección de episodios de hipoxia tisular isquémica mediante la monitorización neurofisiológica intraoperatoria combinada con la monitorización de la oxigenación
} tisular en la cirugía aneurismática

\author{
F. Arikan; J. Vilalta; T. Minoves*; D. Moncho*; A. Vilalta**; M. Noguer***; B. Ibarra**** y J. Sahuquillo
}

Servicios de Neurocirugía, *Neurofisiología, ***Anestesiología y Reanimación, y ****Unidad de Neurorradiología Intervencionista. Hospital Universitario Vall d'Hebron. **Unidad de Investigación de Neurotraumatología y Neurocirugía (UNINN), Institut de Recerca Vall d'Hebron. Universidad Autónoma. Barcelona.

Resumen

Introducción. La neuromonitorización intraoperatoria en la cirugía aneurismática puede ser de gran utilidad para determinar posiciones inadecuadas del clip que ocasionen un compromiso parcial o completo del flujo sanguíneo cerebral en los territorios vasculares irrigados por las arterias relacionadas con el aneurisma. La visualización directa de estas arterias tras la aplicación del clip quirúrgico puede ser insuficiente para detectar esta situación potencialmente deletérea. El conocimiento precoz de esta circunstancia permitiría al neurocirujano corregirla y evitar así la hipoxia tisular cerebral isquémica. Mostramos, con el ejemplo de un caso clínico, la utilidad de la monitorización intraoperatoria de la presión tisular de oxígeno $\left(\mathrm{PtiO}_{2}\right)$ y de los potenciales evocados somatosensoriales (PESS) para la detección de estas situaciones.

Caso clínico. Presentamos el caso de una mujer de 62 años de edad, que debutó con una hemorragia subaracnoidea de origen aneurismático. La arteriografía cerebral demostró la existencia de un aneurisma de la arteria comunicante posterior izquierda que fue tratado inicialmente por vía endovascular con exclusión parcial del aneurisma. Por este motivo se decidió completar el tratamiento mediante cirugía programada. La paciente fue monitorizada intraoperatoriamente con un sensor de $\mathrm{PtiO}_{2}$ situado en el área de riesgo y con PESS. Tras la colocación del clip se produjo una rápida caída de la presión parcial de oxígeno, así como disminución de la amplitud del potencial cortical del nervio tibial posterior izquierdo. El conocimiento de esta situación, permitió detectar un atrapamiento de la arteria comunicante posterior. Tras corregir esta situación reposicionando el clip quirúrgico, ambas variables recuperaron sus valores basales.

Conclusiones. La monitorización intraoperatoria de la $\mathrm{PtiO}_{2}$ combinada con la monitorización neurofisiológica durante la cirugía aneurismática ofrece, de una

Recibido: 19-02-07. Aceptado:6-07-07 forma rápida y fiable, la detección precoz de fenómenos isquémicos ocasionados por mal posicionamiento del clip quirúrgico.

PALABRAS CLAVE: Hemorragia subaracnoidea. Neurocirugía vascular. Monitorización intraoperatoria. Presión tisular de oxígeno. Potenciales evocados somatosensoriales.

Detection of episodes of ischemic tissue hypoxia by means of the combined intraoperative neurophysiologic monitoring with the tissue oxygenation monitoring in aneurysm surgery

\section{Summary}

Introduction. Intraoperative neuromonitoring in aneurysm surgery can be very useful to determine inadequate positions of the vascular clip that cause partial or complete compromise of the cerebral sanguineous flow in the vascular territories irrigated by the arteries related to aneurysm. The direct visualization of these arteries after the application of the surgical clip can be insufficient in detecting this potentially detrimental situation. Knowing this circumstance on the onset would allow the neurosurgeon to correct it and to avoid, therefore, cerebral ischemic tissue hypoxia. We show the utility of the intraoperative monitoring of the oxygen tissue pressure $\left(\mathrm{PtiO}_{2}\right)$ and the somatosensorial evoked potential (SSEP) for the detection of these situations with the example of a clinical case.

Clinical case. We present the case of a 62 year-old woman, that presented with subarachnoid hemorrhage of aneurysmal origin. The cerebral arteriography demonstrated the existence of an aneurysm of the posterior communicating artery that was treated initially by endovascular procedure with partial exclusion of the aneurysm. For this reason it was decided to complete the treatment with a programmed surgery. The patient was put on an intraoperative monitoring system with a $\mathrm{PtiO}_{2}$ sensor located in the risk area and with SSEP. 
After positioning the surgical clip the partial oxygen pressure decreased rapidly, as well as the amplitude of the cortical potential of the left posterior tibial nerve. The knowledge of this situation allowed the detection of a trapped posterior communicating artery. After correcting this situation by replacing the surgical clip, both variables recovered to their basal values.

Conclusions. The intraoperative $\mathrm{PtiO}_{2}$ monitoring, combined with neurophysiologic monitoring during aneurysm surgery offers a fast and trustworthy form of early detection of ischemic phenomena caused by bad positioning of the surgical clip.

KEY WORDS: Subarachnoid hemorrhage. Vascular neurosurgery. Intraoperative monitoring. Oxygen tissue pressure. Somatosensory evoked potentials.

\section{Introducción}

La aparición de acontecimientos isquémicos intraoperatorios o postoperatorios, debidos a la oclusión accidental (parcial o completa) de una o varias de las arterias relacionadas con un aneurisma cerebral, es una de las principales causas de mal pronóstico relacionadas con la propia técnica quirúrgica. La especial atención del cirujano a las arterias relacionadas con el aneurisma durante el clipaje del cuello y la revisión meticulosa de la situación del mismo después de su colocación pueden ser maniobras insuficientes para detectar una reducción significativa del flujo sanguíneo cerebral regional (FSCr) que provoquen lesiones isquémicas postquirúrgicas. Es por tanto importante durante los procedimientos neurovasculares, utilizar técnicas sensibles y específicas que permitan alertar al neurocirujano de forma precoz y fiable de la existencia de estas situaciones isquémicas.

Presentamos un caso práctico, en el que se pone de manifiesto que las técnicas de monitorización intraoperatoria con sensores de presión parcial de oxígeno $\left(\mathrm{PtiO}_{2}\right)$ junto a la monitorización neurofisiológica constituyen métodos sensibles y fiables que permiten detectar de una forma inmediata los fenómenos isquémicos relacionados con estos procedimientos neuroquirúrgicos.

\section{Caso clínico}

La paciente que presentamos, de 62 años de edad, tenía antecedentes de tabaquismo, hipertensión arterial, enfermedad pulmonar obstructiva crónica y depresión mayor. Había sido encontrada por sus familiares inconsciente en su domicilio. En el examen neurológico inicial realizado por el servicio de emergencias médicas (SEM) presentaba una puntuación inicial de cuatro puntos en la escala de coma de Glasgow. La TC cerebral a su ingreso en nuestro centro

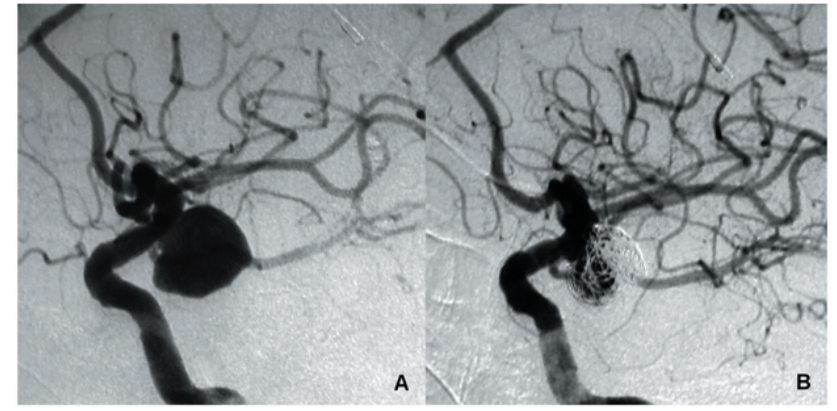

Figura 1. Arteriografía cerebral: A) Estudio angiográfico, carótida interna izquierda: A nivel de la salida de la arteria comunicante posterior izquierda se evidencia la existencia de un aneurisma de $15 \mathrm{~mm}$ de diámetro. La arteria comunicante posterior muestra una buena separación respecto al cuello aneurismático. B) Control angiográfico inmediato que muestra una embolización parcial del aneurisma.

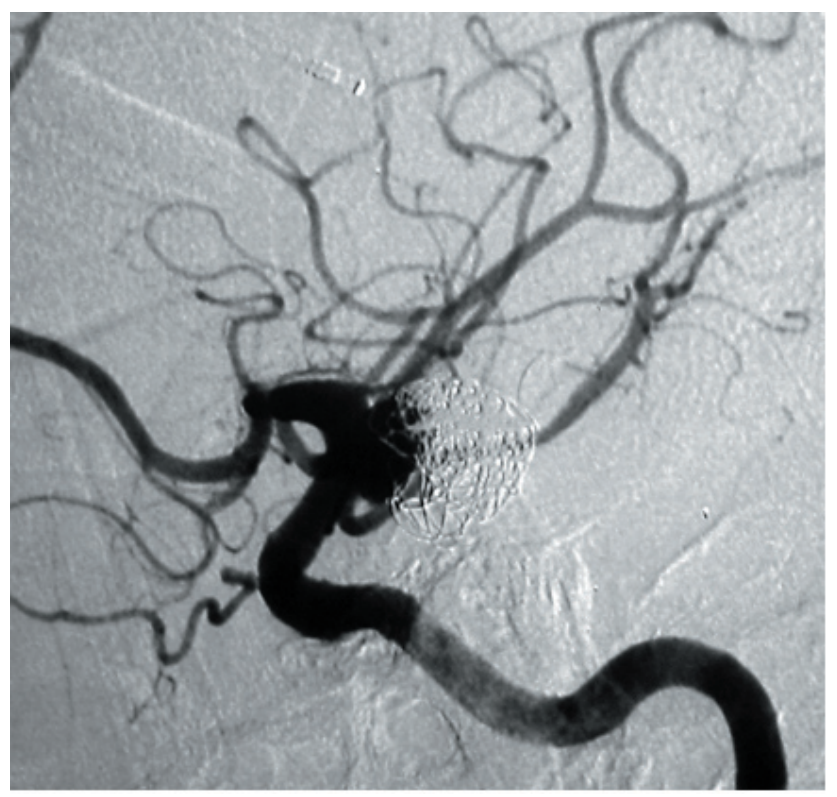

Figura 2. La compactación de los coils a nivel de la cúpula aneurismática permite visualizar perfectamente el cuello del aneurisma.

objetivó la existencia de una hemorragia subaracnoidea masiva, con invasión del sistema ventricular (Grado 4 de Fisher) e hidrocefalia.

El mismo día del ingreso se procedió a la colocación de un drenaje ventricular externo, asociado a un sensor de presión intracraneal tipo Camino (Integra Neurosciences, Plainsboro, NJ, USA) y se le realizó un estudio de angioTC y una arteriografía cerebral. Ambas pruebas objetivaron la presencia de un aneurisma de $16 \mathrm{~mm}$ de diámetro máximo, localizado a nivel de la arteria comunicante posterior izquierda. Dada la mala situación clínica de la enferma y a pesar de las características radiológicas del aneurisma 
se optó por el tratamiento endovascular con el que se consiguió una embolización parcial del aneurisma (Figura 1). Un mes más tarde, mientras permanecía todavía en la unidad de cuidados intensivos, consciente, parcialmente desorientada y sin déficits neurológicos focales, se realizó una arteriografía cerebral de control. La paciente era todavía portadora de un drenaje ventricular externo con un débito alto de LCR. En el nuevo estudio se observó la oclusión parcial del aneurisma que presentaba una compactación de los coils en la cúpula aneurismática, visualizándose sin embargo, la permeabilidad del cuello del aneurisma (Figura 2). Dada la evolución clínica favorable, se decidió la intervención quirúrgica para la exclusión completa del aneurisma.

\section{Estudio neurofisiológico preoperatorio}

Se le practicaron potenciales evocados somatosensoriales (PESS) y potenciales evocados auditivos de tronco cerebral (PEATC) el día previo a la cirugía. Presentaba unas respuestas neurofisiológicas de características adecuadas para la monitorización intraoperatoria de PEATC y de PESS.

\section{Intervención quirúrgica}

El día 4 de noviembre de 2005 fue intervenida de forma electiva bajo anestesia general. A la llegada de la enferma a quirófano se procedió a la monitorización no invasiva (ECG, TA incruenta y pulsioximetria), seguidamente se realizó la inducción anestésica con fentanilo (150 $\mu \mathrm{gr})$, propofol (175 $\mathrm{mg}$ ) y atracurio (30mg). La intubación se realizó mediante un tubo anillado de $7,5 \mathrm{~mm}$. de diámetro iniciándose ventilación en modo control de volumen con una $\mathrm{FiO}_{2}$ de $0,62 \%$, un VT 600cc y una FR de 9/minuto. Una vez anestesiada, se monitorizó la temperatura esofágica, la capnografía y la diuresis. Se procedió a la cateterización de la arteria radial izquierda para el control de la TA y la vena basílica izquierda para el control de la PVC. En el momento de la fijación del cabezal se inyectaron $300 \mu \mathrm{g}$ de fentanilo y 50 $\mathrm{mg}$ de propofol. El mantenimiento anestésico se realizó con remifentanilo endovenoso a $0,1-0,2 \mu \mathrm{g} \cdot \mathrm{K}^{1-} \min ^{1-}$ y desflurano al 3\%. Se procedió a la monitorización intraoperatoria neurofisiológica con PESS y PEATC, y monitorización intraoperatoria de la $\mathrm{PtiO}_{2}$. Durante la intervención se evitaron los bolus de fármacos y el mantenimiento anestésico se realizó sin perfusión de relajante muscular. Los parámetros respiratorios se modificaron según gasometrías, manteniendo siempre una $\mathrm{PaCO}_{2}$ de alrededor de $35 \mathrm{mmHg}$. La paciente se mantuvo estable durante toda la intervención.

\section{Monitorización intraoperatoria neurofisiológica}

La monitorización intraoperatoria se realizó utilizando un aparato de registro con programa de monitorización intraoperatoria Keypoint ${ }^{\circledR}$ de 4 canales y estimulador dual (Medtronic A/S, Copenhagen, Denmark). Durante la cirugía se monitorizó de forma continua la integridad funcional de las vías sensitivas, mediante el estudio de los PESS de ambos nervios tibiales posteriores. La estimulación se efectuó mediante electrodos de superficie situados en zona retromaleolar interna. Los registros se realizaron a nivel cortical en Cz-Fpz según el sistema internacional 10-20. Este sistema 10-20 es el método más ampliamente utilizado para describir la localización de los electrodos subcutáneos en el cuero cabelludo. Este método se basa en la relación entre la localización de un electrodo y el área de córtex cerebral subyacente. Cada sitio tiene una letra (para identificar la región cerebral) y un número u otra letra para identificar la localización hemisférica. Las letras usadas son: "F" para el lóbulo Frontal, "T" para el temporal , "C" para el vertex, "P" para el parietal y, "O" para el lóbulo occipital. Los números pares $(2,4,6,8)$ se refieren al hemisferio derecho y los impares $(1,3,5,7)$ al izquierdo. "Z" se refiere a los electrodos colocados en la línea media. El número menor, es el más cercano a la posición en línea media. "Fp" es el Fronto-polar. "Nasion" es el punto entre la frente y la nariz. "Inion" es la protuberancia occipital externa.

La monitorización de la vía auditiva se efectuó mediante una estimulación binaural alternante con clics a una intensidad de $105 \mathrm{~dB}$. El registro se realizó en la zona retroauricular bilateral usando como referencia $\mathrm{Cz}$. Las respuestas basales fueron obtenidas una vez el paciente estuvo anestesiado. Estas respuestas demostraron la adecuada morfología y reproducibilidad para ser usadas como referencia y compararlas con las obtenidas durante la cirugía. Los PESS basales del nervio tibial posterior derecho presentaban una amplitud P1-N1 de $0.5 \mu \mathrm{V}$ y una latencia del componente P1 de 38,2 ms. Los PESS izquierdos mostraban una amplitud P1-N1 de $0.5 \mu \mathrm{V}$ y una latencia P1 de 38,1 ms. Los PEATC objetivaron en estudio basal una latencia de $6 \mathrm{~ms}$ de la onda $\mathrm{V}$ derecha y de $5,9 \mathrm{~ms}$ para la izquierda.

\section{Monitorización intraoperatoria de la presión parcial de oxígeno}

Dada la presencia de una circulación fetal se consideró como área de riesgo isquémico el territorio de la arteria cerebral posterior izquierda. Con el objetivo de detectar de una forma precoz un posible atrapamiento de la arteria comunicante posterior durante el clipaje del aneurisma y para la monitorización continua intraoperatoria de la $\mathrm{PtiO}_{2}$, se colocó antes de iniciar la cirugía un catéter polarográfico tipo Clark CC1.2 (Licox GMS, Kiel, Alemania) a nivel subcortical occipital izquierdo mediante la realización de 


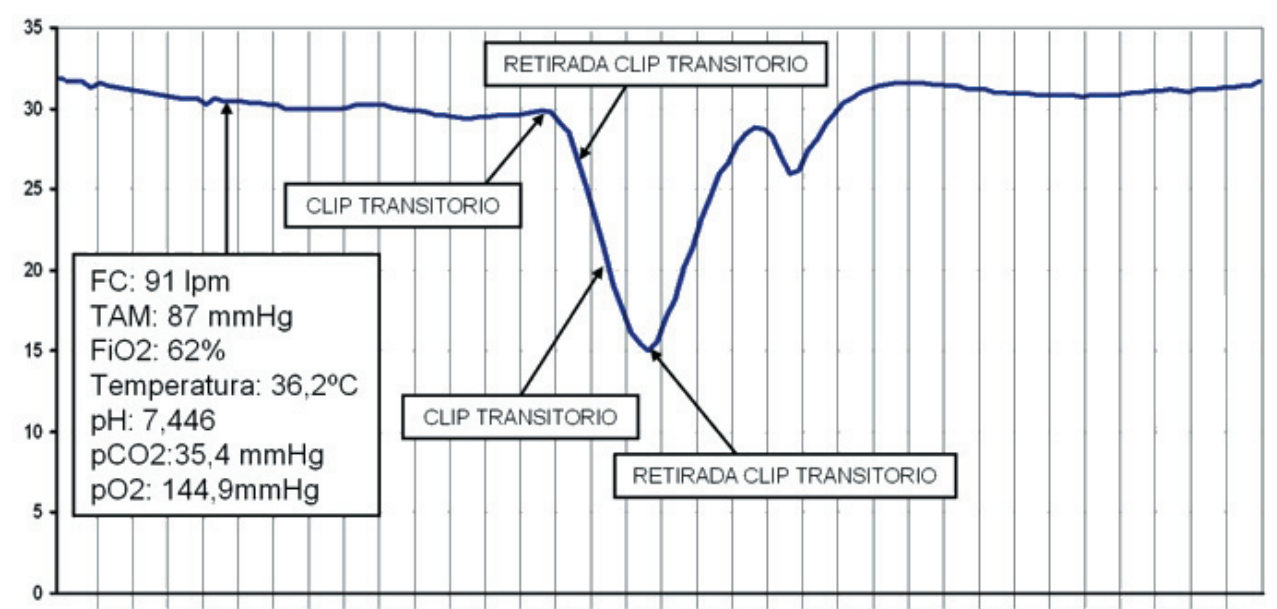

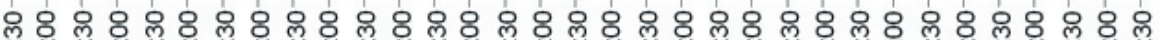
هั

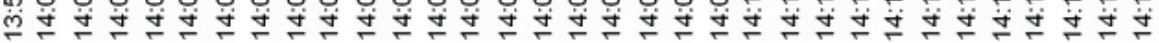

Figura 3. Registro de la presión tisular de oxígeno (PtiO). Después de 45 minutos, la PtiO, se estabilizó en valores entre 30 - $35 \mathrm{mmHg}$ (valores considerados de referencia). Durante el clipaje transitorio se produjo una caída de la $\mathrm{PtiO}_{2}$ que persisitió tras la retirada del clip transitorio por lo que se procedió a explorar el clip definitivo. Tras la segunda retirada del clip transitorio se evidenció una recuperación rápida en las cifras de $\mathrm{PtiO}_{2}$.

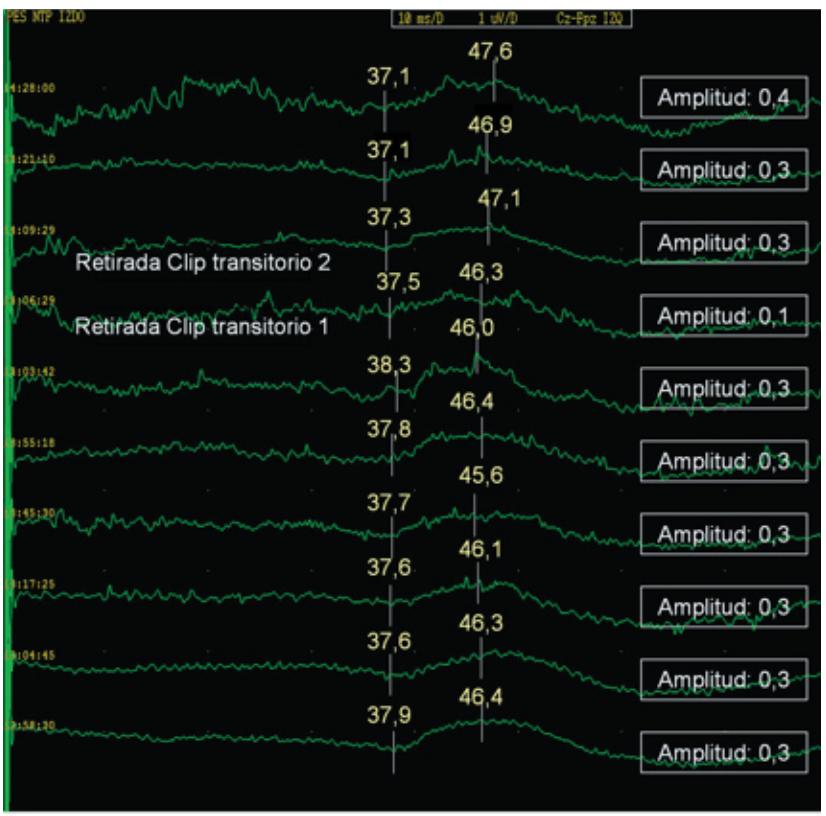

Figura 4. Monitorización neurofisiológica intraoperatoria correspondiente a los PESS corticales de nervio tibial posterior izquierdo. Obsérvese la caída en la amplitud del potencial tras la retirada del primer clip transitorio y su recuperación una vez retirado el segundo clip transitorio.

un pequeño orificio de craneostomía realizado a $5 \mathrm{~cm}$ por encima del inión y a $3 \mathrm{~cm}$ de la línea media. Tras un tiempo de 45 minutos, la $\mathrm{PtiO}_{2}$ se estabilizó mostrando una meseta entre los 30 y $35 \mathrm{mmHg}$. En el momento de esta lectura, el valor de las variables sistémicas eran las siguientes: frecuencia cardiaca de $91 \mathrm{pm}, \mathrm{TAM}$ de $87 \mathrm{mmHg}, \mathrm{FiO}_{2}$ del $62 \%$, temperatura esofágica de $36,2^{\circ} \mathrm{C}, \mathrm{pH}$ de $7,44, \mathrm{pCO}_{2}$ de $35,4 \mathrm{mmHg}$ y $\mathrm{PaO}_{2}$ de $144,9 \mathrm{mmHg}$. Estos valores fueron los considerados como referencia.

\section{Procedimiento quirúrgico}

Para el abordaje del aneurisma se realizó una craneotomía fronto-orbitaria izquierda realizada en un solo bloque. Tras la abertura de la porción proximal del valle Silviano y de las cisternas basales se procedió a la disección del aneurisma para mostrar sus relaciones anatómicas. Dado el tamaño del aneurisma, la arteria comunicante posterior no podía ser visualizada sin desplazar el aneurisma. A continuación se procedió a la colocación de un clip transitorio recto de $9 \mathrm{~mm}$ en la carótida izquierda supraclinoidea para facilitar la colocación del clip definitivo a nivel del cuello del aneurisma.

\section{Resultados de la monitorización intraoperatoria (Figuras 3 y 4)}

Durante el clipaje transitorio se produjo una disminución significativa de la amplitud del potencial cortical así como una caída progresiva de la $\mathrm{PtiO}_{2}$. Tras la retirada del clip transitorio se evidenció como la $\mathrm{PtiO}_{2}$ continuaba descendiendo, apareciendo además una disminución significativa de la amplitud del potencial cortical al estimular el nervio tibial posterior del lado izquierdo (de $0.3 \mu \mathrm{V}$ a 0,1 $\mu \mathrm{V}$ ). Dada la concordancia de ambas alteraciones, se procedió a re-explorar la colocación de clip, observándose un atrapamiento de la arteria comunicante posterior. Se procedió de nuevo al clipaje transitorio de la carótida izquierda y a la reposición del clip definitivo, con especial atención a la arteria comunicante posterior (Figura 5). Tras la segunda retirada del clip transitorio se evidenció una recuperación rápida en las cifras de $\mathrm{PtiO}_{2}$ cuyo valor mínimo había sido de $14 \mathrm{mmHg}$. Tras la recolocación del clip se produjo también, una recuperación a los valores previos de la amplitud del potencial cortical del nervio tibial posterior del lado 


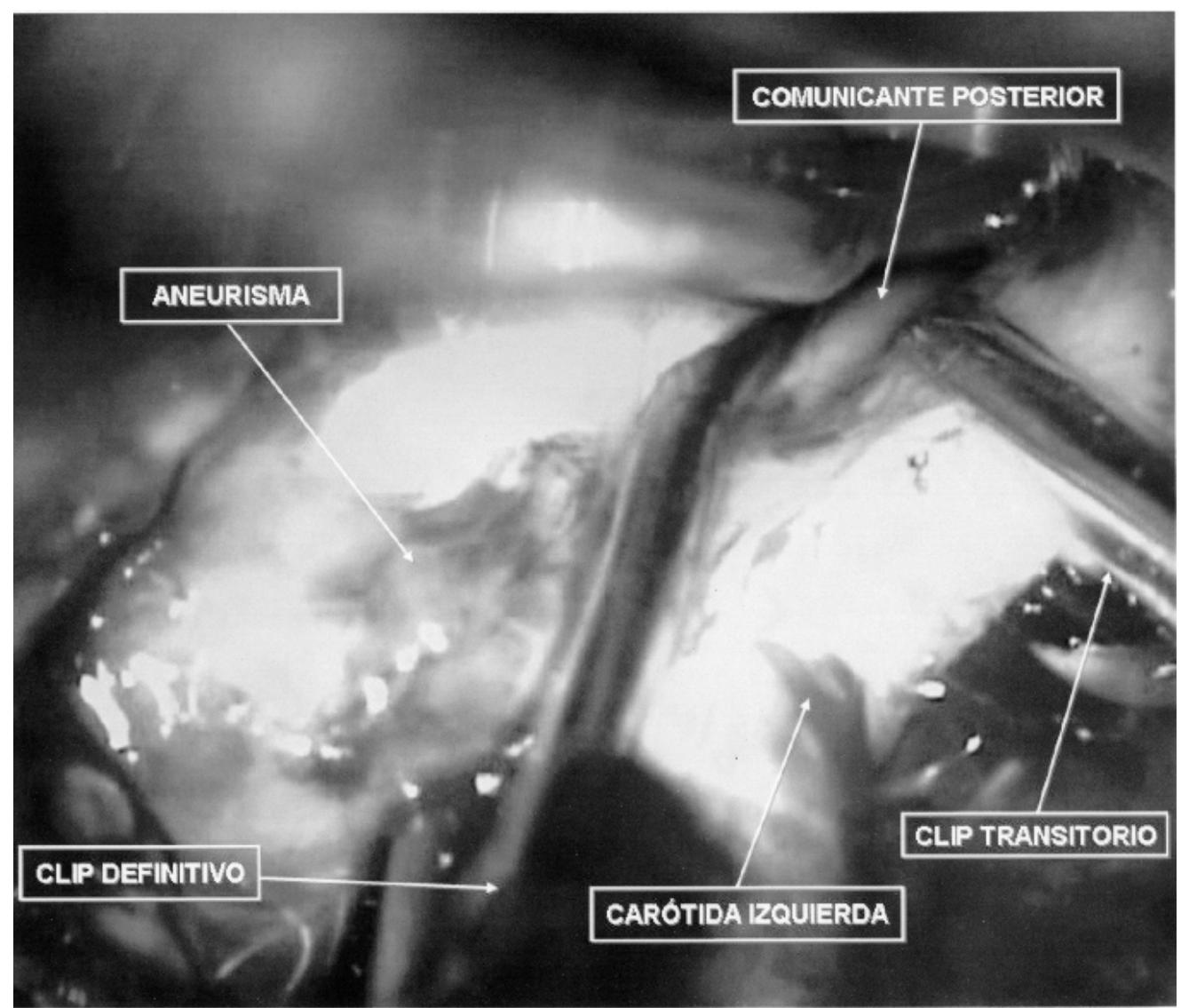

2008; 19: $113-120$

Figura 5. Imagen de la cirugía que muestra el reposicionamiento del clip definitivo con especial atención a la arteria comunicante posterior.

izquierdo. Hubo un tiempo de isquemia para el territorio de la arteria cerebral posterior de un minuto y medio (tiempo entre la colocación del primer clip transitorio y la retirada del segundo). En ningún momento se observaron cambios significativos en el PESS de nervio tibial posterior del lado derecho ni en los PEAT bilaterales.

\section{Evolución postoperatoria}

El curso postoperatorio transcurrió sin incidencias clínicas relevantes. Las TC de control no evidenciaron la presencia de lesiones isquémicas. Dado el largo periodo de ingreso y encamamiento, tras la implantación de una válvula de derivación ventrículo-peritoneal de LCR, la paciente fue trasladada sin focalidades neurológicas a un centro de rehabilitación.

\section{Discusión}

El objetivo principal en la cirugía aneurismática es la de excluir el aneurisma de la circulación cerebral preservando el flujo a través de las arterias relacionadas a este. La aparición de lesiones isquémicas postoperatorias por oclusiones parciales o totales de una o varias de estas arterias constituye una de las principales causas de mal pronóstico rela- cionadas directamente con la técnica quirúrgica. A pesar de que el abordaje microquirúrgico permite una inspección visual directa, ésta ha mostrado ser en muchos casos insuficiente para detectar compromisos parciales o totales del flujo. En la literatura se recogen porcentajes que oscilan de 6 a $31 \%$ de oclusiones o estenosis inadvertidas por la simple inspección visual y reveladas mediante la realización de arteriografías intraoperatorias o postoperatorias, o mediante el uso del dopplers microvasculares intraoperator $\operatorname{ios}^{1,4,29,35}$.

A pesar de considerarse la arteriografía como el patrón oro (Gold Standard) para el diagnóstico de compromisos de flujo en las arterias relacionadas con el saco aneurismático, esta técnica no puede ser realizada de forma rutinaria en la mayoría de centros neuroquirúrgicos de nuestro entorno por falta de medios técnicos y personales. Además, aunque diferentes autores han demostrado la utilizad de la arteriografía intraoperatoria para el diagnóstico y la corrección en el mismo acto quirúrgico de los rellenos aneurismáticos residuales, el tiempo necesario para llevar a término esta técnica diagnóstica no permite un resultado inmediato, siendo por lo tanto controvertida su utilidad para evitar la aparición de lesiones isquémicas postquirúrgicas $^{1,12,14,35,36}$.

En estas cirugías es por lo tanto recomendable dispo- 
ner de sistemas de monitorización continuos y fiables que permitan la detección en tiempo real de compromisos de flujo que ocasionen situaciones de isquemia cerebral regional. La monitorización de la presión tisular de oxígeno ha demostrado ser un sistema de detección fiable, inmediato y de fácil interpretación para la detección de situaciones de hipoxia cerebral isquémica. Sin embargo, presenta como principal inconveniente el hecho de que se trata de un sistema de monitorización regional. Es muy importante por tanto, en este tipo de patología, en donde el objetivo es detectar situaciones de hipoxia debidas a fenómenos isquémicos causados por una mal posición del clip quirúrgico, una correcta selección del territorio o territorios vasculares a monitorizar, puesto que si monitorizamos un territorio diferente al de la arteria comprometida, este sistema sería incapaz de detectarlo ${ }^{18,24,25,37}$. En nuestro caso consideramos el territorio de la arteria cerebral posterior izquierda como la principal área de riesgo, debido a que en esta paciente este territorio dependía únicamente del sistema carotídeo. Una oclusión inadvertida por el clip quirúrgico de la arteria comunicante posterior ocasionaría un infarto en este territorio vascular. Sin embargo, con el uso de un solo sensor seríamos incapaces de detectar compromisos en otros territorios vasculares (p.e. a nivel de la coroidea anterior). Este problema puede paliarse en parte, mediante la colocación de más de un sensor de $\mathrm{PtiO}_{2}$, o bien mediante la asociación de la $\mathrm{PtiO}_{2}$ con un sistema de monitorización más global, como es la monitorización intraoperatoria neurofisiológica.

La monitorización neurofisiológica intraoperatoria constituye una técnica que nos informa sobre el estado funcional de regiones cerebrales específicas de una forma global, constituyendo al mismo tiempo una medida indirecta de hipoxia tisular isquémica. Esta técnica, al igual que la $\mathrm{PtiO}_{2}$, permite una monitorización en tiempo real, detectando de una forma precoz los potenciales eventos deletéreos. Dentro de las diferentes técnicas de monitorización neurofisiológica intraoperatoria, los PESS y PEATC son los más ampliamente utilizados en la patología vascular cerebral debido a la correlación existente entre los fenómenos de isquemia o daño cerebral y las alteraciones electrofisiológicas. Estas alteraciones, a nivel de la cirugía aneurismática, pueden correlacionarse con compromisos vasculares ocasionados por el clip quirúrgico, vasoespasmo o incluso por uso excesivo de retractores. Tanto en modelos de experimentación animal como en la literatura neuroquirúrgica, se ha establecido la relación existente entre los cambios en la monitorización neurofisiológica y los cambios regionales del flujo sanguíneo cerebral ${ }^{2,5-11,15,20-22,27,28,39-}$ 42,47. Cabe destacar también, que en los últimos años se está extendiendo el uso de los potenciales evocados motores dentro de las técnicas de monitorización neurofisiológica intraoperatoria, ya que éstos han demostrado tener una ele- vada sensibilidad en la detección de fenómenos isquémicos en la cirugía aneurísmática ${ }^{23,34,45}$. Nosotros en nuestro caso, dada la disponibilidad en nuestro centro, aplicamos únicamente la monitorización de los PESS y PEATC. De hecho, ambos sistemas de monitorización detectaron de forma inmediata y simultánea a la retirada del clip transitorio el compromiso existente a nivel de la arteria comunicante posterior.

Mediante el uso combinado de PESS y PEATC conseguimos una valoración global de todo el encéfalo puesto que los PESS permiten una monitorización de las distribuciones vasculares de la arteria cerebral media, anterior, posterior y carótida interna, y los PEATC la monitorización de la circulación posterior $3,8,13,16,17,19,26,28,30-33,38,43,44,46$.

Otra de las ventajas que ofrece la monitorización combinada de $\mathrm{PtiO}_{2}$ y monitorización neurofisiológica, es la de prever una vez solucionado el evento isquémico, la posible repercusión clínica que éste haya podido tener. A nivel de oximetría tisular, es conocido que valores de $\mathrm{PtiO}_{2}$ inferiores a $8 \mathrm{mmHg}$. mantenidos más de 30 minutos son predictivos de la aparición de un infarto cerebral ${ }^{25}$. En nuestro caso, ni el valor mínimo de $8 \mathrm{mmHg}$ alcanzado, ni el tiempo total del evento (4 minutos) repercutieron en la aparición de una lesión isquémica postoperatoria. Del mismo modo, en nuestro caso, la recuperación a los valores basales del potencial sensitivo fueron indicativos de una buena recuperación funcional.

\section{Conclusiones}

La monitorización intraoperatoria de la presión parcial de oxígeno $\left(\mathrm{PtiO}_{2}\right)$ como medida de detección regional combinada con la monitorización intraoperatoria neurofisiológica como medida de detección global durante la cirugía aneurismática ofrece, de una forma rápida y fiable, la detección precoz de fenómenos isquémicos ocasionados por posicionamientos inadecuados e inadvertidos del clip quirúrgico.

\section{Bibliografía}

1. Alexander, T.D., Macdonald, R.L., Weir, B., et al: Intraoperative angiography in cerebral aneurysm surgery: a prospective study of 100 craniotomies. Neurosurgery 1996; 39: 10-17.

2. Anderson, L.C., Hemler, D.E., Luethke, J.M., et al: Transcranial magnetic evoked potentials used to monitor the spinal cord during neuroradiologic angiography of the spine. Spine 1994; 19: 613-616.

3. Aravabhumi, S., Izzo, K.L., Bakst, B.L.: Brainstem auditory evoked potentials: intraoperative monitoring technique in surgery of posterior fossa tumors. Arch Phys Med Rehabil 1987; 68: 142-146. 
4. Bailes, J.E., Tantuwaya, L.S., Fukushima, T., et al.: Intraoperative microvascular Doppler sonography in aneurysm surgery. Neurosurgery 1997; 40: 965-970.

5. Branston, N.M., Ladds, A., Symon, L., et al.: Comparison of the effects of ischaemia on early components of the somatosensory evoked potential in brainstem, thalamus, and cerebral cortex. J Cereb Blood Flow Metab 1984; 4: 68-81.

6. Branston, N.M., Strong, A.J., Symon, L.: Extracellular potassium activity, evoked potential and tissue blood flow. Relationships during progressive ischaemia in baboon cerebral cortex. J Neurol Sci 1997; 32: 305-321.

7. Branston, N.M., Symon, L., Crockard, H.A., et al.: Relationship between the cortical evoked potential and local cortical blood flow following acute middle cerebral artery occlusion in the baboon. Exp Neurol 1974; 45: 195-208.

8. Buchthal, A., Belopavlovic, M.: Somatosensory evoked potentials in cerebral aneurysm surgery. Klin Wochenschr 66 Suppl 1988; 14: 27-34.

9. Buchthal, A., Belopavlovic, M.: Somatosensory evoked potentials in cerebral aneurysm surgery. Eur J Anaesthesiol 1992; 9: 493-497.

10. Buchthal, A., Belopavlovic, M., Mooij, J.J.: Evoked potential monitoring and temporary clipping in cerebral aneurysm surgery. Acta Neurochir (Wien ) 1988; 93: 28-36.

11. Cloughesy, T.F., Nuwer, M.R., Hoch, D., et al.: Monitoring carotid test occlusions with continuous EEG and clinical examination. J Clin Neurophysiol 1993; 10: 363-369.

12. Derdeyn, C.P., Moran, C.J., Cross, D.T., III, et al.: Intracranial aneurysm: anatomic factors that predict the usefulness of intraoperative angiography. Radiology 1997; 205: 335-339.

13. Djuric, S., Milenkovic, Z., Klopcic-Spevak, M., et al.: Somatosensory evoked potential monitoring during intracranial surgery. Acta Neurochir (Wien ) 1992; 119: 85-90.

14. Fagundes-Pereyra, W.J., Hoffman, W.E., Misra, M., et al.: Clip readjustment in aneurysm surgery after flow evaluation using the ultrasonic perivascular probe: case report. Arq Neuropsiquiatr 2005; 63: 339-344.

15. Ferbert, A., Buchner, H., Bruckmann, H., et al.: Evoked potentials in basilar artery thrombosis: correlation with clinical and angiographic findings. Electroencephalogr Clin Neurophysiol 1988; 69: 136-147.

16. Friedman, W.A., Chadwick, G.M., Verhoeven, F.J., et al.: Monitoring of somatosensory evoked potentials during surgery for middle cerebral artery aneurysms. Neurosurgery 1991; 29: 83-88.

17. Friedman, W.A., Kaplan, B.L., Day, A.L., et al.: Evoked potential monitoring during aneurysm operation: observations after fifty cases. Neurosurgery 1987; 20: 678-687.

18. Gelabert-González, M., Fernández-Villa, J.M., Ginesta-Galán, V.: Intra-operative monitoring of brain tissue O2 (PtiO2) during aneurysm surgery. Acta Neurochir (Wien ) 2002; 144: 863-866.
19. Grundy, B.L., Nelson, P.B., Lina, A., et al.: Monitoring of cortical somatosensory evoked potentials to determine the safety of sacrificing the anterior cerebral artery. Neurosurgery 1982; 11: 64-67.

20. Hacke, W., Berg-Dammer, E., Zeumer, H.: Evoked potential monitoring during acute occlusion of the basilar artery and selective local thrombolytic therapy. Arch Psychiatr Nervenkr 1983; 232: 541-548.

21. Hacke, W., Zeumer, H., Berg-Dammer, E.: Monitoring of hemispheric or brainstem functions with neurophysiologic methods during interventional neuroradiology. AJNR Am J Neuroradiol 1983; 4: 382-384.

22. Hacke, W., Zeumer, H., Ringelstein, E.B.: EEG controlled occlusion of the internal carotid artery during angiography. Neuroradiology 1981; 22: 19-22.

23. Horiuchi, K., Suzuki, K., Sasaki, T., et al.: Intraoperative monitoring of blood flow insufficiency during surgery of middle cerebral artery aneurysms. J Neurosurg 2005; 103: 275-283.

24. Ibáñez, J., Vilalta, A., Mena, M.P., et al.: [Intraoperative detection of ischemic brain hypoxia using oxygen tissue pressure microprobes]. Neurocirugía 2003; 14: 483-489.

25. Kett-White, R., Hutchinson, P.J., Al Rawi, P.G., et al.: Cerebral oxygen and microdialysis monitoring during aneurysm surgery: effects of blood pressure, cerebrospinal fluid drainage, and temporary clipping on infarction. J Neurosurg 2002; 96: 1013-1019.

26. Little, J.R., Lesser, R.P., Luders, H.: Electrophysiological monitoring during basilar aneurysm operation. Neurosurgery 1987; 20: 421-427.

27. Liu, A.Y., López, J.R., Do, H.M., et al.: Neurophysiological monitoring in the endovascular therapy of aneurysms. AJNR Am J Neuroradiol 2003; 24: 1520-1527.

28. López, J.R., Chang, S.D., Steinberg, G.K.: The use of electrophysiological monitoring in the intraoperative management of intracranial aneurysms. J Neurol Neurosurg Psychiatry 1999; 66: 189-196.

29. Macdonald, R.L., Wallace, M.C., Kestle, J.R.: Role of angiography following aneurysm surgery. J Neurosurg 1993; 79: 826-832.

30. Manninen, P.H., Patterson, S., Lam, A.M., et al.: Evoked potential monitoring during posterior fossa aneurysm surgery: a comparison of two modalities. Can J Anaesth 1994; 41: 92-97.

31. McPherson, R.W., Niedermeyer, E.F., Otenasek, R.J., et al.: Correlation of transient neurological deficit and somatosensory evoked potentials after intracranial aneurysm surgery. Case report. J Neurosurg 1983; 59: 146-149.

32. Mizoi, K., Yoshimoto, T.: Permissible temporary occlusion time in aneurysm surgery as evaluated by evoked potential monitoring. Neurosurgery 1993; 33: 434-440.

33. Momma, F., Wang, A.D., Symon, L.: Effects of temporary arterial occlusion on somatosensory evoked responses in 
aneurysm surgery. Surg Neurol 1987; 27: 343-352.

34. Neuloh, G., Schramm, J.: Monitoring of motor evoked potentials compared with somatosensory evoked potentials and microvascular Doppler ultrasonography in cerebral aneurysm surgery. J Neurosurg 2004; 100: 389-399.

35. Origitano, T.C., Schwartz, K., Anderson, D., et al.: Optimal clip application and intraoperative angiography for intracranial aneurysms. Surg Neurol 1999; 51: 117-124.

36. Payner, T.D., Horner, T.G., Leipzig, T.J., et al.: Role of intraoperative angiography in the surgical treatment of cerebral aneurysms. J Neurosurg 1998; 88: 441-448.

37. Poca, M.A., Sahuquillo, J., Mena, M.P., et al.: [Recent advances in regional cerebral monitoring in the neurocritical patient: brain tissue oxygen pressure monitoring, cerebral microdialysis and near-infrared spectroscopy.]. Neurocirugía 2005; 16: 385-410.

38. Schramm, J., Koht, A., Schmidt, G., et al.: Surgical and electrophysiological observations during clipping of 134 aneurysms with evoked potential monitoring. Neurosurgery 1990; 26: 61-70.

39. Sharbrough, F.W., Messick, J.M., Jr., Sundt, T.M., Jr.: Correlation of continuous electroencephalograms with cerebral blood flow measurements during carotid endarterectomy. Stroke 1973; 4: 674-683.

40. Sundt, T.M., Jr., Michenfelder, J.D.: Focal transient cerebral ischemia in the squirrel monkey. Effect on brain adenosine triphosphate and lactate levels with electrocorticographic and pathologic correlation. Circ Res 1972; 30: 703-712.

41. Sundt, T.M., Jr., Sharbrough, F.W., Anderson, R.E., et al.: Cerebral blood flow measurements and electroencephalograms during carotid endarterectomy. J Neurosurg 1974; 41 : $310-320$.
42. Sundt, T.M., Jr., Sharbrough, F.W., Piepgras, D.G., et al.: Correlation of cerebral blood flow and electroencephalographic changes during carotid endarterectomy: with results of surgery and hemodynamics of cerebral ischemia. Mayo Clin Proc 1981; 56: 533-543.

43. Symon, L., Momma, F., Murota, T.: Assessment of reversible cerebral ischaemia in man: intraoperative monitoring of the somatosensory evoked response. Acta Neurochir Suppl (Wien ) 1988; 42: 3-7.

44. Symon, L., Wang, A.D., Costa e Silva, I.E., et al.: Perioperative use of somatosensory evoked responses in aneurysm surgery. J Neurosurg 1984; 60: 269-275.

45. Szelenyi, A., Langer, D., Kothbauer, K., et al.: Monitoring of muscle motor evoked potentials during cerebral aneurysm surgery: intraoperative changes and postoperative outcome. J Neurosurg 105: 675-681.

46. Wagner, W., Peghini-Halbig, L., Maurer, J.C., et al.: Intraoperative SEP monitoring in neurosurgery around the brain stem and cervical spinal cord: differential recording of subcortical components. J Neurosurg 1994; 81: 213-220.

47. Zentner, J., Schumacher, M., Bien, S.: Motor evoked potentials during interventional neuroradiology. Neuroradiology 1988; 30: 252-255.

Arikan, F.; Vilalta, J.; Minoves, T.; Moncho, D.; Vilalta, A.; Noguer, M.; Ibarra, B.; Sahuquillo, J.: Detección de episodios de hipoxia tisular isquémica mediante la monitorización neurofisiológica intraoperatoria combinada con la monitorización de la oxigenación tisular en la cirugía aneurismática. Neurocirugía 2008; 19: 113-120.

Correspondencia postal: Fuat Arikan Abelló. C/ Mora de Ebro 65-67, A, 2-4. 08023 Barcelona. 